\title{
Perfil clínico-epidemiológico de pacientes oncológicos na região do Bolsão Sul-Matogrossense
}

\section{Clinical-epidemiological profile of cancer patients in the Bolsão Sul-Matogrossense Region}

Rachid Figueirôa Souza, Antonio Carlos Cavalcante Godoy, Mirian Daiane de Oliveira, Aline Rafaela da Silva Rodrigues Machado, Alex Martins Machado

\section{RESUMO}

Objetivo: Determinar se a região atendida pela Unidade de Assistência de Alta Complexidade em Oncologia de Três Lagoas (UNACON - TL), referência oncológica no Bolsão Sul-Matogrossense, apresenta semelhança com a média geral de casos de neoplasias sólidas do Brasil e do estado, além de evidenciar, através de associações entre as variáveis coletadas, possíveis fatores que exerçam influência sobre 0 óbito na região. Métodos: Estudo epidemiológico de delineamento transversal, baseado em prontuários, incluindo pacientes diagnosticados com neoplasias sólidas, entre janeiro de 2008 e dezembro de 2017. O perfil epidemiológico foi avaliado através das variáveis: sexo, residência, faixa etária, raça, CID-10, ano de diagnóstico, estádio, diagnóstico, tratamento e evolução, utilizando método da Quasi-Verossimilhança. Resultados: Foram identificados 1.760 casos neste período, podendo observar que a região tem semelhança com a média geral nacional e do estado. Com as associações, ficou demonstrado que a proximidade e 0 acesso ao serviço especializado diminuíram a mortalidade. Além disso, chamou a atenção a verificação de que os indivíduos mais jovens tiveram desfechos mais desfavoráveis, além de uma significativa mortalidade na raça branca. No mais, constatou-se que a taxa de sobrevida é mais alta quanto menor o estadiamento na região. Considerações Finais: A pesquisa, apesar de confirmar a semelhança com a média geral brasileira e do estado, pode estabelecer correlações importantes entre as variáveis e o desfecho óbito, chamando atenção para o aspecto heterogêneo da região estudada, trazendo implicações para a gestão e a prática assistencial de que é preciso buscar a qualificação e sustentabilidade dos sistemas de informação sobre câncer, para que os resultados obtidos criem os meios adequados para enfrentar os desafios e subsidiem o desenvolvimento de ações eficazes para a saúde pública no Brasil.

PALAVRAS-CHAVE: Epidemiologia; Neoplasias; Funções verossimilhança; Perfil da saúde.

\section{ABSTRACT}

Objective: To determine if the region served by the Unit of High Complexity Assistance in Oncology of Três Lagoas (HCAO - TL), an oncological reference in the Bolsão Sul-Matogrossense region, has similarity with the general average of cases of solid neoplasms in Brazil and the state, in addition to showing, through associations between the collected variables, possible factors that influence death in the region. Methods: Epidemiological cross-sectional study, based on medical records, including patients diagnosed with solid neoplasms, between January 2008 and December 2017. The epidemiological profile was assessed using the variables: sex, residence, age, race, ICD-10, year of diagnosis, stage, diagnosis, treatment and evolution, using the Quasi-Likelihood method. Results: Were identified, in this period, 1,760 cases, showing that the region is similar to the national and state general average. With the associations, it has been demonstrated that proximity and access to specialized services reduces mortality. In addition, attention was drawn to the finding that younger individuals had more unfavorable outcomes, in addition to significant mortality in the white race. In addition, it was found that the survival rate is higher the lower the staging in the region. Final Considerations: The research, despite confirming the similarity with the general Brazilian and state average, can establish important correlations between the variables and the death outcome, drawing attention to the heterogeneous aspect of the studied region, bringing implications for management and care practice that it is necessary to seek the qualification and sustainability of cancer information systems, so that the results obtained create the appropriate means to face the challenges and support the development of effective actions for public health in Brazil. 


\section{INTRODUÇÃO}

As transições demográficas e epidemiológicas globais sinalizam um impacto cada vez maior da carga de câncer nas próximas décadas ${ }^{1}$. Estima-se, para o Brasil, no biênio 2020-2022, a ocorrência de 625 mil casos novos de câncer para cada ano. Excetuando-se o câncer de pele não melanoma (cerca de 170 mil casos novos), ocorrerão 450 mil casos novos de câncer. 0 cálculo global corrigido para o sub-registro aponta a ocorrência de 640 mil casos novos².

No estado de Mato Grosso do Sul, o padrão observado é de altas taxas de incidência e tendência de incremento, de modo geral, seguindo o comportamento observado para o país². Porém, a composição bastante heterogênea das macrorregiões do estado provoca questionamentos quanto às variações regionais na incidência de neoplasias sólidas. Além disso, sabe-se que informações sobre incidência também são afetadas por diferenças na capacidade diagnóstica dos serviços de saúde, o que pode levar a uma subestimação da incidência real em algumas regiões.

Com isso, e levando em consideração a inexistência de estudos que analisem os dados regionais do Bolsão Sul-Matogrossense, há o despertar do interesse em realizar uma avaliação nesta região, legitimada pela recente habilitação da Unidade de Assistência de Alta Complexidade em Oncologia (UNACON) em Três Lagoas ${ }^{3,4}$.

Dessa forma, este estudo teve por objetivo determinar se a região atendida pela Unidade de Assistência de Alta Complexidade em Oncologia de Três Lagoas (UNACON - TL), referência oncológica no Bolsão Sul-Matogrossense, apresenta semelhança com a média geral de casos de neoplasias sólidas do Brasil e do estado, além de evidenciar, através de associações entre as variáveis coletadas, possíveis fatores que exerçam influência sobre o óbito na região.

\section{MÉTODO}

Foi realizado um estudo epidemiológico com delineamento transversal, baseado na análise de prontuários do Unidade de Assistência de Alta Complexidade em Oncologia de Três Lagoas (UNACON - TL), referência para a região do Bolsão Sul-Matogrossense em diagnóstico e tratamento de neoplasias. O estudo foi aprovado pelo Comité de Ética em Pesquisa Humana da Universidade Federal de Mato Grosso do Sul, sob o número: 1.672 .084 de 10 de agosto de 2016.

Foram incluídos os pacientes diagnosticados com neoplasias sólidas, cuja localização primária se encontra classificada no índice C da Classificação Estatística Internacional de Doenças e Problemas Relacionados à Saúde Décima Revisão (CID 10)5, e acompanhados pela UNACON de Janeiro de 2008, ano de inicio das atividades do serviço de oncologia, até Dezembro de 2017.

O banco de dados foi formado por 1.760 casos, possuindo medidas variáveis categóricas de caracterização, informações relativas a sexo, residência, faixa etária, raça, estádio, meio de diagnóstico, evolução para óbito, forma de tratamento e grupo (tipo de neoplasia). 
As informações sobre a incidência dos tipos de neoplasias nacionais e estaduais foram obtidas por intermédio dos dados disponibilizados pelo Instituto Nacional do Câncer (INCA) e os dados populacionais através do Instituto Brasileiro de Geografia e Estatística (IBGE) ${ }^{6}$.

Para avaliar o número de casos de neoplasias da região e ajustá-lo entre as variáveis escolhidas foi utilizada uma Regressão Log-Linear ${ }^{6}$. Para verificar os fatores que exerciam influência sobre a ocorrência do óbito foi utilizada uma Regressão Logística ${ }^{7}$, sendo o método Backward ${ }^{8}$ utilizado para seleção de variáveis.

Para comparar a incidência de atendimentos de neoplasias em Três Lagoas e no Brasil; em Três Lagoas e no Mato Grosso do Sul foram utilizados os bancos de dados do INCA e do IBGE. Sendo assim, para avaliar o comportamento da proporção de neoplasias gerais e específicas ao longo do tempo foi utilizado o Modelo de Regressão de Poisson.

O modelo de Poisson considera a variância igual a média, o qual pode ocasionar o fenômeno de sub ou super dispersão. Portanto, nesse trabalho foi utilizado o método da Quasi-Verossimilhança ${ }^{7,9}$ para a estimação do modelo. 0 software utilizado nas análises foi o R (versão 3.5.2).

\section{RESULTADOS}

A descrição dos casos coletados durante o período de análises, evidenciando as variáveis de caracterização utilizadas, é exposta na tabela 1.

Tabela 1. Análise descritiva das variáveis de caracterização.

\begin{tabular}{llll}
\hline Variáveis & Categorias & Freq. Absoluta & Freq. Relativa \\
\hline Sexo & Feminino & 908 & $51,58 \%$ \\
& Masculino & 852 & $48,42 \%$ \\
\hline \multirow{2}{*}{ Residência } & Três Lagoas & 1195 & $67,89 \%$ \\
& Microrregião de Três Lagoas & 69 & $3,91 \%$ \\
& Mesorregião do Leste de MS & 104 & $5,90 \%$ \\
& Outras cidades do MS & 302 & $17,16 \%$ \\
& Outros Estados & 90 & $5,13 \%$ \\
\hline \multirow{5}{*}{ Faixa etária } & De 15 a 19 anos & 4 & $0,23 \%$ \\
& De 20 a 29 anos & 18 & $1,00 \%$ \\
& De 30 a 39 anos & 77 & $4,37 \%$ \\
& De 40 a 49 anos & 170 & $9,66 \%$ \\
& De 50 a 59 anos & 341 & $19,39 \%$ \\
& De 60 a 69 anos & 477 & $27,13 \%$ \\
& De 70 a 79 anos & 413 & $23,45 \%$ \\
& Acima de 80 anos & 260 & $14,79 \%$ \\
\hline
\end{tabular}




\begin{tabular}{|c|c|c|c|}
\hline \multirow[t]{4}{*}{ Raça } & Branco & 553 & $31,44 \%$ \\
\hline & Amarelo & 12 & $0,69 \%$ \\
\hline & Pardo & 1101 & $62,58 \%$ \\
\hline & Preto & 93 & $5,29 \%$ \\
\hline \multirow[t]{2}{*}{ SUS } & Não & 3 & $0,15 \%$ \\
\hline & Sim & 1757 & $99,85 \%$ \\
\hline \multirow[t]{4}{*}{ Estádio } & 1 & 226 & $12,84 \%$ \\
\hline & 2 & 442 & $25,14 \%$ \\
\hline & 3 & 384 & $21,83 \%$ \\
\hline & 4 & 707 & $40,18 \%$ \\
\hline Meio de & Clínico & 134 & $7,61 \%$ \\
\hline \multirow[t]{5}{*}{ diagnóstico } & Marcadores tumorais & 16 & $0,93 \%$ \\
\hline & Citologia & 19 & $1,10 \%$ \\
\hline & Histologia da metástase & 31 & $1,78 \%$ \\
\hline & Histologia do tumor primário & 1485 & $84,35 \%$ \\
\hline & Exames radiológicos & 74 & $4,23 \%$ \\
\hline \multirow[t]{5}{*}{ Tratamento } & Não & 112 & $6,37 \%$ \\
\hline & Quimioterapia & 465 & $26,40 \%$ \\
\hline & Radioterapia & 59 & $3,38 \%$ \\
\hline & Cirúrgico & 963 & $54,72 \%$ \\
\hline & Quimio e Radio & 161 & $9,13 \%$ \\
\hline Evoluiu para & Não & 1341 & $76,21 \%$ \\
\hline óbito & Sim & 419 & $23,79 \%$ \\
\hline \multirow[t]{17}{*}{ Sítio primário } & Bexiga & 51 & $2,87 \%$ \\
\hline & Cavidade Oral & 101 & $5,74 \%$ \\
\hline & Colo do útero & 96 & $5,46 \%$ \\
\hline & Cólon e reto & 192 & $10,91 \%$ \\
\hline & Corpo do útero & 17 & $0,98 \%$ \\
\hline & Esôfago & 49 & $2,81 \%$ \\
\hline & Estômago & 82 & $4,65 \%$ \\
\hline & Glândula tireóide & 32 & $1,84 \%$ \\
\hline & Laringe & 34 & $1,95 \%$ \\
\hline & Mama feminina & 270 & $15,34 \%$ \\
\hline & Melanoma maligno da pele & 3 & $0,17 \%$ \\
\hline & Outras neoplasias malignas da pele & 352 & $19,99 \%$ \\
\hline & Ovário & 20 & $1,15 \%$ \\
\hline & Próstata & 176 & $9,99 \%$ \\
\hline & Sistema nervoso central & 4 & $0,23 \%$ \\
\hline & Traquéia, brônquio e pulmão & 113 & $6,43 \%$ \\
\hline & Outros* & 167 & $9,48 \%$ \\
\hline
\end{tabular}




\begin{tabular}{llll} 
Ano de & 2008 & 175 & $9,94 \%$ \\
diagnóstico & 2009 & 142 & $8,04 \%$ \\
& 2010 & 124 & $7,06 \%$ \\
2011 & 77 & $4,37 \%$ \\
2012 & 89 & $5,05 \%$ \\
2013 & 117 & $6,66 \%$ \\
2014 & 237 & $13,44 \%$ \\
2015 & 274 & $15,57 \%$ \\
2016 & 270 & $15,34 \%$ \\
\hline
\end{tabular}

* Demais neoplasias sólidas cuja localização primária se encontra classificada no índice C do CID 10.

\section{Comparação da incidência de neoplasias entre os locais}

De forma a responder o primeiro objetivo do estudo, foi realizada a comparação entre as incidências de neoplasias sólidas atendidas na UNACON-TL com os dados nacionais e do estado de Mato Grosso do Sul. (Figura 2).

Figura 2 - Comparação da incidência de neoplasias da região estudada com as incidências de neoplasias do estado de Mato Grosso do Sul e as incidências nacionais.
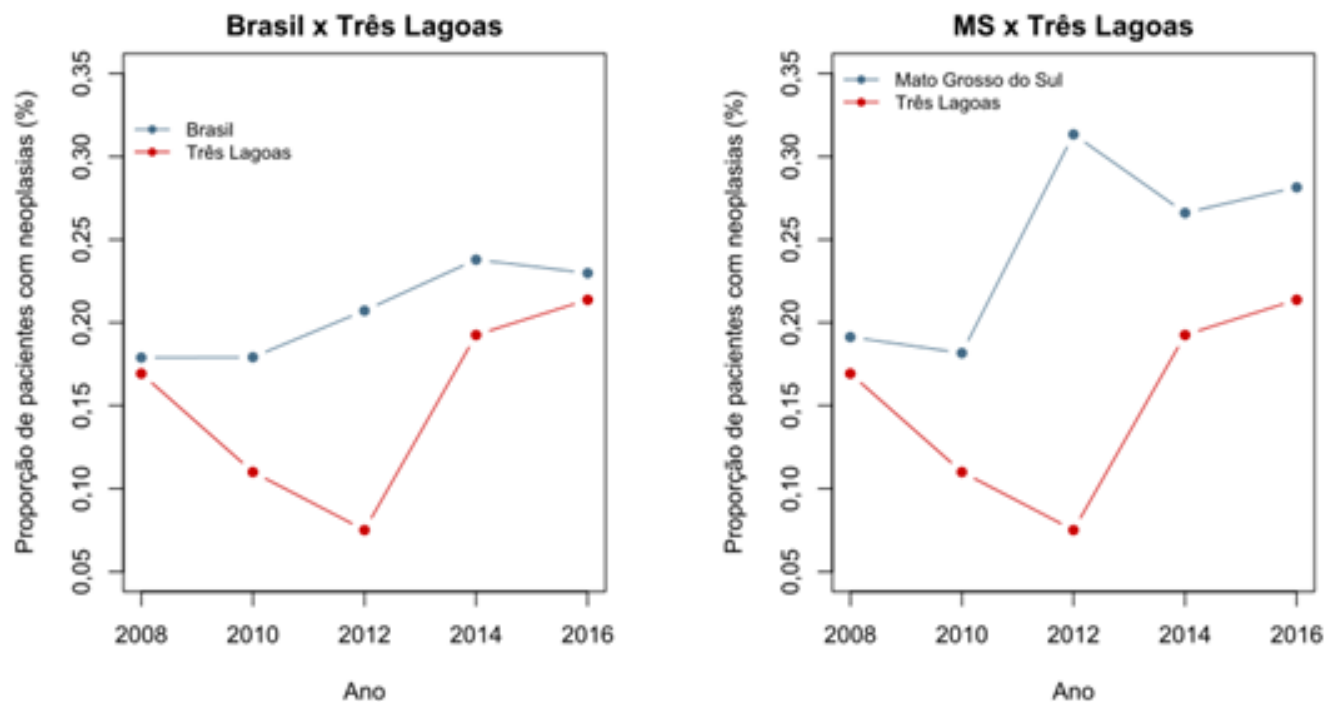

A partir das comparações das incidências das neoplasias em geral, pode-se verificar que:

- Não houve diferença significativa $(p=0,946)$ do comportamento da proporção de pacientes com neoplasias sólidas ao longo do tempo entre os índices locais e os nacionais (UNACON-TL x Brasil).

- Não houve diferença significativa $(p=0,938)$ do comportamento da proporção de pacientes com neoplasias sólidas ao longo do tempo entre os índices locais e os estaduais (UNACON-TL x Mato Grosso do Sul 


\section{Fatores que exercem influência sobre o óbito na Região}

Somado ao fato da ocorrência dos cânceres ser de etiologia multifatorial, podendo ter origem na combinação de vários fatores - genéticos, ambientais e de modos de vida, sabe-se que o desfecho dessas doenças sofre influência por diferenças nas condições de acesso, uso e desempenho dos serviços de saúde - componentes importantes das condições de vida da população.

Um sinergismo perigoso que potencializa o risco de desenvolvimento e de óbito de determinados tipos dos cânceres, podendo a redução isolada de apenas um fator ser insuficiente para garantir a efetividade de ações de prevenção.

Diante desse sinergismo, e dispondo dos dados coletados nesse estudo, realizamos associações entre as variáveis e o desfecho óbito, com o objetivo de identificar possivveis fatores que exerçam influência sobre o óbito na região estudada.

\section{Análise univariada}

Inicialmente foi realizada uma análise univariada das variáveis sexo, residência, faixa etária, raça, estádio e tratamento com relação à ocorrência de óbito. (Tabela 2)

Tabela 2. Análise univariada das variáveis explicativas.

\begin{tabular}{|c|c|c|c|c|c|c|}
\hline \multirow[t]{2}{*}{ Variáveis } & \multirow[t]{2}{*}{ Categorias } & \multicolumn{2}{|c|}{ Óbito $=$ Não } & \multicolumn{2}{|c|}{ Óbito $=\mathrm{Sim}$} & \multirow[b]{2}{*}{ Valor-p } \\
\hline & & $\mathrm{N}$ & $\%$ & $\mathbf{N}$ & $\%$ & \\
\hline \multirow[t]{2}{*}{ Residência } & Outras & 469 & $83,05 \%$ & 96 & $16,95 \%$ & $<0,001^{1}$ \\
\hline & Três Lagoas & 872 & $72,96 \%$ & 323 & $27,04 \%$ & \\
\hline \multirow[t]{4}{*}{ Faixa etária } & De 15 a 29 anos & 12 & $56,25 \%$ & 10 & $43,75 \%$ & $0,003^{2}$ \\
\hline & De 30 a 49 anos & 179 & $72,53 \%$ & 68 & $27,47 \%$ & \\
\hline & De 50 a 69 anos & 658 & $80,40 \%$ & 160 & $19,60 \%$ & \\
\hline & Acima de 70 anos & 492 & $73,09 \%$ & 181 & $26,91 \%$ & \\
\hline \multirow[t]{3}{*}{ Raça } & Branco & 453 & $81,86 \%$ & 100 & $18,14 \%$ & $0,003^{1}$ \\
\hline & Pardo e amarelo & 815 & $73,21 \%$ & 298 & $26,79 \%$ & \\
\hline & Preto & 73 & $78,26 \%$ & 20 & $21,74 \%$ & \\
\hline \multirow[t]{2}{*}{ Próstata } & Não & 1179 & $74,46 \%$ & 405 & $25,54 \%$ & $<0,001^{1}$ \\
\hline & Sim & 157 & $89,47 \%$ & 19 & $10,53 \%$ & \\
\hline \multirow[t]{2}{*}{ Estádio } & 1 e 2 & 621 & $92,98 \%$ & 47 & $7,02 \%$ & $<0,001^{1}$ \\
\hline & 3 e 4 & 661 & $60,59 \%$ & 430 & $39,41 \%$ & \\
\hline \multirow[t]{5}{*}{ Tratamento } & Não & 70 & $62,65 \%$ & 42 & $37,35 \%$ & $<0,001^{1}$ \\
\hline & Radioterapia & 43 & $72,09 \%$ & 16 & $27,91 \%$ & \\
\hline & Quimioterapia & 281 & $60,47 \%$ & 184 & $39,53 \%$ & \\
\hline & Quimio e Radio & 113 & $70,34 \%$ & 48 & $29,66 \%$ & \\
\hline & Cirúrgico & 833 & $86,54 \%$ & 130 & $13,46 \%$ & \\
\hline
\end{tabular}

${ }^{1}$ Teste Qui-Quadrado. ${ }^{2}$ Teste Exato de Fisher 
A partir da análise realizada, pode-se destacar as seguintes análise:

- Associação significativa $(p<0,001)$ entre município de residência e óbito, sendo que entre os indivíduos que moram em Três Lagoas, 72,96\% não vieram a óbito.

- Associação significativa $(p=0,003)$ entre faixa etária e óbito, sendo que entre os indivíduos com idade entre 15 e 29 anos, 43,75\% vieram a óbito. Em comparação na faixa entre 50 e 69 anos, apenas 19,6\% evoluíram para óbito.

- Associação significativa ( $p=0,003$ ) entre raça e óbito, sendo que entre os brancos, $18,14 \%$ vieram a óbito, em detrimento de $26,79 \%$ de pardos e amarelos.

- Associação significativa $(p<0,001)$ entre neoplasias de próstata e óbito, sendo que entre indivíduos que tiveram a neoplasia, somente $10,53 \%$ vieram a óbito.

- Associação significativa ( $p<0,001)$ entre estádio e óbito, sendo que entre indivíduos em estádio 1 e 2, 92,98\% não evoluíram para óbito. Em comparação com 60,59\% dos estádios 3 e 4.

\section{Análise multivariada}

Foram selecionadas para a análise multivariada aquelas que apresentaram um valor-p inferior a 0,25 na análise univariada. Desta forma, procedeu-se com uma análise multivariada usando modelos multivariados inicial e final para a ocorrência de óbito. (Tabela 3)

Tabela 3. Ajuste multivariado das variáveis explicativas

\begin{tabular}{llllllll}
\hline Variáveis Categorias & O.R. & I.C. $(95 \%)$ & Valor-p & $\mathbf{0 . R}$. & I.C. $(95 \%)$ & Valor-p \\
\hline Sexo & Feminino & 1,00 & - & - & - & - & - \\
& Masculino & 0,99 & {$[0,69 ; 1,41]$} & 0,947 & - & - & - \\
\hline Residência & Três Lagoas & 1,00 & - & - & 1,00 & - & - \\
& Outras & 0,50 & {$[0,35 ; 0,71]$} & $<0,001$ & 0,50 & {$[0,35 ; 0,70]$} & $<0,001$ \\
\hline \multirow{2}{*}{ Faixa etária } & De 15 a 29 anos & 1,00 & - & - & - & - & - \\
& De 30 a 49 anos & 0,59 & {$[0,17 ; 2,09]$} & 0,411 & - & - & - \\
& De 50 a 69 anos & 0,41 & {$[0,12 ; 1,39]$} & 0,152 & - & - & - \\
& Acima de 70 anos & 0,78 & {$[0,23 ; 2,73]$} & 0,703 & - & - & - \\
\hline \multirow{2}{*}{ Raça } & Branco & 1,00 & - & - & - & - & - \\
& Pardo e Amarelo & 1,22 & {$[0,86 ; 1,75]$} & 0,269 & - & - & - \\
& Preto & 0,90 & {$[0,44 ; 1,85]$} & 0,777 & - & - & - \\
\hline \multirow{2}{*}{ Estádio } & 1 e 2 & 1,00 & - & - & 1,00 & - & - \\
& 3 e 4 & 6,02 & {$[3,8 ; 9,52]$} & $<0,001$ & 6,65 & {$[4,30 ; 10,29]$} & $<0,001$ \\
\hline \multirow{2}{*}{ Tratamento } & Não & 1,00 & - & - & 1,00 & - & - \\
& Radioterapia & 0,61 & {$[0,23 ; 1,62]$} & 0,322 & 0,58 & {$[0,22 ; 1,50]$} & 0,258 \\
& Quimioterapia & 0,61 & {$[0,32 ; 1,16]$} & 0,132 & 0,56 & {$[0,30 ; 1,04]$} & 0,065 \\
& Quimio e Radio & 0,54 & {$[0,26 ; 1,14]$} & 0,106 & 0,44 & {$[0,22 ; 0,90]$} & 0,025 \\
& Cirúrgico & 0,34 & {$[0,18 ; 0,65]$} & 0,001 & 0,29 & {$[0,16 ; 0,55]$} & $<0,001$ \\
\hline
\end{tabular}


Assim, a partir desta análise, algumas variáveis mostraram-se significativas, como as mostradas abaixo:

- A variável residência foi significativa $(p<0,001)$ sobre a ocorrência do evento óbito; sendo que se a residência não for em Três Lagoas, a chance de óbito diminui 50\% [30\%;65\%].

- O estádio foi significativo $(p<0,001)$ sobre a ocorrência do óbito, sendo que se o estádio for 3 e 4 , a chance de óbito aumenta $565 \%$ [330\% e 929\% respectivamente].

- O tratamento foi significativo sobre a ocorrência do óbito, sendo que se o tratamento for cirúrgico as chances de óbito reduzem $71 \%$ [ $45 \%$ e $84 \%$ respectivamente].

\section{DISCUSSÃO}

Conseguiu-se demonstrar que a região atendida pela UNACON - TL apresenta semelhança com a média geral de casos de neoplasias sólidas do Brasil e do estado.

Apesar dessa conclusão tornar a região homogênea, é importante deixar claro a possível influencia das mudanças ocorridas no serviço, durante o período analisado, no resultado obtido, onde o serviço oncológico passou de um atendimento em menor escala, restrito a uma população já internada, para um complexo multicêntrico e multidisciplinar focado no atendimento específico, deixando de lado o caráter auxiliar, tornando-se um centro de referência em diagnóstico e busca por tratamento.

\section{Fatores que exercem influência sobre o óbito em Três Lagoas}

Apesar de não haver uma demonstração do mecanismo causal das neoplasias sólidas, as associações realizadas nesse estudo revelaram resultados interessantes do ponto de vista prático na saúde pública, identificando componentes modificáveis que podem ser suficientes para grandes avanços na prevenção, a partir da escolha de medidas preventivas.

\section{Análise univariada}

Quanto à associação entre residência e óbito, a residência em Três Lagoas demonstrou uma alta taxa, 72,96\%, de sobrevida, evidenciando que a proximidade e o acesso ao serviço especializado diminui a mortalidade em função da melhora e rapidez no atendimento ${ }^{15}$.

A alta taxa de óbito, 43,75\%, na faixa etária de 15 a 29 anos pode ser justificada por uma evolução mais desfavorável das neoplasias em jovens. Carcinomas mamários, pulmonares e colorretais em pacientes jovens geralmente apresentam características clínicas e patológicas associadas à doença mais agressiva, apresentando comportamento clínico, patológico e perfil imunofenotípico de maior agressividade biológica, cursando com pior prognóstico e menores sobrevidas global ${ }^{16,17}$. 
$\mathrm{Na}$ associação entre raça e óbito, observamos que na raça branca, $18,14 \%$ vieram a óbito. Apesar de muitos estudos mostrarem que os indivíduos de ascendência negra têm um risco globalmente aumentado de malignidades em comparação com os caucasianos ${ }^{18}$, nosso estudo mostrou uma significativa mortalidade na raça branca. É preciso explorar ainda mais a contribuição dos fatores biológicos para as diferenças raciais no risco de câncer e mortalidade. Porém, sem esquecer do papel dos determinantes socioambientais, como o acesso aos serviços de saúde.

Ainda nesta análise, observou-se que dos pacientes em estádio 1 e 2, mostraram alta taxa de sobrevida (92,98\%). Assim, constata-se que um dos fatores que influenciam fortemente a sobrevida é a extensão da doença no momento do diagnóstico, mostrando que um diagnóstico rápido pode ser essencial para uma maior sobrevida ${ }^{19}$.

\section{Análise multivariada}

Diferentemente do observado anteriormente, nos estadiamentos iniciais, quando observado estadiamentos mais tardios (3 e 4), a chance de óbito aumentou 565\% [330\% e 929\% respectivamente]. Isto é um reflexo do que ocorre no Brasil, porém em uma escala maior. A auditoria operacional realizada pelo Tribunal de Contas da União em 2010 identificou que $60,5 \%$ dos pacientes com câncer foram diagnosticados já em estadiamentos avançados (níveis 3 e 4). $O$ diagnóstico tardio foi identificado como uma das principais barreiras enfrentadas pelos pacientes no acesso ao tratamento, seja pela falta de centros especializados, pelas longas esperas para consultas com médicos especialistas ou pela demora na realização de exames ${ }^{15}$.

Finalmente, quando analisados os fatores de tratamento, o tratamento cirúrgico mostrou uma melhor redução das taxas de óbito com 71\% [45\%;84\%]. A cirurgia é apropriada para tratamento da doença localizada e regional, além de poder curar os estádios mais iniciais.

É importante frisar que os não tratados são representados por aqueles que optam por livre e espontânea vontade ou por não terem condição clínica de serem submetidos a um tratamento. Ou seja, apesar do tratamento cirúrgico ter demonstrado melhor redução, este possui um papel muito limitado nos estádios avançados, aumentando os índices de óbito nestes indivíduos².

Dessa forma, fica evidente, através dos dados obtidos pelas correlações, o quão importante é se ter uma melhor compreensão de uma região, muito além de uma simples análise comparativa entre incidências e, consequentemente, caracterização de forma homogênea, sem se levar em conta o comportamento de suas variáveis.

Desse modo, a partir do conhecimento das associações entre variáveis apresentadas no estudo, é visível que ações isoladas e homogêneas de prevenção, tratamento e cura serão pouco efetivas na região, e que o sucesso na redução da mortalidade passa necessariamente pela melhora das condições do contexto local, ou seja, o estabelecimento de ações de prevenção primária levando em conta as características da região. E para tal, é de suma importância a constante alimentação dessas ações com a manutenção de um sistema de vigilância com informações oportunas e de qualidade que subsidie análises epidemiológicas para as tomadas de decisões. 


\section{CONSIDERAÇÕES FINAIS}

Nosso estudo, com seus achados, em uma região até então inexplorada, apesar de confirmar a semelhança com a média geral brasileira e do estado de casos de neoplasias sólidas, pode estabelecer correlações importantes entre as variáveis e o desfecho óbito, chamando atenção para o aspecto heterogêneo da região estudada.

Trazendo implicações para a gestão e a prática assistencial de que é preciso buscar a qualificação e sustentabilidade dos sistemas de informação sobre câncer, assim como demais sistemas em saúde, para que os resultados obtidos criem os meios adequados para enfrentar os desafios e subsidiem o desenvolvimento de ações eficazes para a saúde pública no Brasil.

Além disso, reitera-se que as informações geradas com esse estudo sejam utilizadas não somente para a gestão, como também para a percepção da população no enfrentamento das neoplasias, promovendo um olhar além do método técnico, servindo como um catalisador, que estimule gestores, profissionais de saúde, pesquisadores, comunicadores e a sociedade em geral para a reflexão acerca da questão.

\section{REFERÊNCIAS}

1. Ferlay J, et al. Cancer today. Lyon, France: International Agency for Research on Cancer, 2018. (IARC CAncerBase, n. 15). Disponivel em: https://publications.iarc.fr/Databases/larc-Cancerbases/Cancer-Today-Powered-By-GLOBOCAN-2018--2018. Acesso em: Setembro 2020.

2. Brasil. Ministério da Saúde. Secretaria de Atenção à Saúde. Instituto Nacional de Câncer. Coordenação de Prevenção e Vigilância. Estimativa 2020: incidência de câncer no Brasil. Rio de Janeiro: INCA, 2020.

3. Instituto Nacional de Câncer José Alencar Gomes da Silva. Informativo Detecção Precoce, Boletim ano 7, n. 1, janeiro/abril de 2016. Disponível em: https://www.inca.gov.br/publicacoes/informativos/informativo-deteccao-precoce-no-1-2016. Acesso em: Agosto 2019.

4. Brasil. Ministério da Saúde. Portaria nº 149/ GM, de 20 de Fevereiro de 2013. Habilita estabelecimento como Unidade de Assistência de Alta Complexidade em Oncologia (UNACON). Diário Oficial da União, Brasília, DF. 2013. 
5. Organização Mundial da Saúde. Classificação Estatística Internacional de Doenças e Problemas Relacionados à Saúde: CID-10. Décima revisão. Trad de Centro Colaborador da OMS para a Classificação de Doenças em Português. v. 2, 3 ed. São Paulo: EDUSP, 1996.

6. Instituto Brasileiro de Geografia e Estatística (IBGE). Censo demográfico 2010. Conceitos e métodos. Rio de Janeiro: IBGE, 2011. Disponível em: https://www.ibge.gov.br/estatisticas/sociais/populacao/9662-censo-demografico-2010.html?=\&t=conceitose-metodos. Acesso em: Fevereiro 2020.

7. Mccullagh P, Nelder JA. Generalized Linear Models. [s.I.] CRC press, 1989.

8. $\quad$ Agresti A. Categorical data analysis. Hoboken. New Jersey: John Wiley \& Sons Inc. 2012; 267-313.

9. Weddeburn R. Quasi like lihood functions, generalized linear models and the Gauss-Newton method. Biometrika. 1974; 7: 439-444.

10. Ministério da Saúde. Secretaria de Vigilância em Saúde. Departamento de Análise de Situação de Saúde. Plano de ações estratégicas para o enfrentamento das doenças crônicas não transmissíveis (DCNT) no Brasil 20112022. Brasília. 2011.

11. Malta DC, Silva JrJB. Plano de Ações Estratégicas para o Enfrentamento das Doenças Crônicas Não Transmissíveis no Brasil após três anos de implantação, 2011-2013. Epidemiol Serv Saúde. 2014; 23(3): 389-98.

12. Brasil. Ministério da Saúde. Portaria n 874/ GM, de 16 de Maio de 2013. Institui a Política Nacional para a Prevenção e Controle do Câncer na Rede de Atenção à Saúde das Pessoas com Doenças Crônicas no âmbito do Sistema Único de Saúde (SUS). Diário Oficial da União, Brasília, DF. 2013; Seção 1: 129-132.

13. Litwin MS, Tan H. The Diagnosis and Treatment of Prostate CancerA Review. JAMA. 2017; 317(24): 2532-2542.

14. Thun MJ et al. Cancer epidemiology and prevention. 4th ed. New York: Oxford University Press, 2017. 
15. Tribunal de Contas da União. Relatório de Auditoria Operacional na Política Nacional de Atenção Oncológica (número 031.944/2010-8) [Internet]. 2010. Disponível em:http://portal2.tcu.gov.br/portal/page/portal/TCU/comunidades/programas_governo/areas_atuacao/saude/Oncologia-relatório-versão final.pdf. Acesso em: Março 2020.

16. American Cancer Society. Cancer facts \& figures 2019. Atlanta: American Cancer Society, 2019.

17. Bray, F. et al. Global cancer statistics 2018: GLOBOCAN estimates of incidence and mortality worldwide for 36 cancers in 185 countries. CA: a cancer journal for clinicians, Hoboken, v. 68, n. 6, p. 394-424, Nov. 2018.

18. Islami F. et al. Proportion and number of cancer cases and deaths attributable to potentially modifiable risk factors in the United States. CA: a cancer journal for clinicians, Hoboken. 2018; 68(1): 31-54.

19. Instituto Nacional de Câncer José Alencar Gomes da Silva. Tipos de câncer. Rio de Janeiro: INCA, 2019. Disponível em: https://www.inca.gov.br/tipos-de-cancer. Acesso em: Setembro 2019. 